\title{
Autonomía profesional y riesgos de seguridad de los periodistas en Colombia
}

\author{
Miguel E. Garcés Prettel, Jesús Arroyave Cabrera*
}

Perfiles Latinoamericanos

Flacso México

\begin{abstract}
Resumen
Este artículo analiza la relación entre autonomía profesional y los riesgos de seguridad de los periodistas en Colombia, a partir de una investigación correlacional-transversal que utilizó un muestreo por cuota de 751 periodistas que diligenciaron a nivel nacional el cuestionario del estudio "Worlds of Journalism". Como resultado se encontró que la variable "agresiones recibidas" presentó diferencias significativas respecto al género, la sección informativa, la región, el medio informativo, los años de experiencia, el cargo y el nivel educativo de los periodistas; que las agresiones correlacionan positivamente con la autonomía para publicar noticias sobre gobiernos, fuerzas armadas, bandas criminales y problemas sociales estructurales (pobreza, situación de las minorías étnicas, desigualdad social, daños ambientales), y que este último es un factor predictor del riesgo alto de seguridad. La autonomía mostró ser un factor protector del acoso sexual laboral y del arresto ilegal.
\end{abstract}

\begin{abstract}
This paper analyzes the relationship between professional autonomy and security risks of journalists in Colombia. A correlational-transversal research was conducted with a sample of 751 journalists who filled out the questionnaire "Worlds of Journalism Study". The results show significant differences on the attacks received by the journalists depending on gender, news beat, region, news media, years of experience, capacity and educational level of journalists. Attacks on journalists correlates positively with the autonomy to publish and write news on governments, armed forces, criminal gangs and structural social problems (poverty, status of ethnic minorities, socioeconomic inequality, environmental damage) the latter being a predictor of high risk security.
\end{abstract}

Palabras clave: periodismo, agresiones, riesgo, autonomía profesional, noticias, libertad de prensa, seguridad.

Keywords: Journalism, attacks, risk, professional autonomy, news, press freedom, security.

* Magister en Comunicación Social y candidato a doctor en Comunicación de la Universidad del Norte (Colombia). Profesor asociado, Universidad Tecnológica de Bolívar (Colombia).

** Doctor en Comunicación por la Universidad de Miami (Estados Unidos). Profesor asociado, Universidad del Norte (Colombia). 


\section{Introducción ${ }^{1}$}

periodismo visibiliza acontecimientos noticiosos desde una perspectiva informativa y analítica o involucrando denuncias sobre problemas que afectan a la sociedad, lo que convierte a los periodistas en actores sociales influyentes que generan opinión pública (Mellado, 2014), y al oficio como tal, en una labor de alto riesgo para sus vidas (Weaver \& Wilhoit, 1996; Canel \& Sadaba, 1999; Ortega \& Humanes, 2000; Martini \& Luchesi, 2004).

El tema del riesgo en el periodismo se ha abordado tradicionalmente desde las teorías de comunicación de riesgo para referirse tanto a las coberturas informativas frente a la gestión de desastres naturales (Miralles, 2009; Obregón, Arroyave \& Barrios, 2009; 2010) como al tipo de información que requiere la opinión pública en circunstancias de peligro, crisis o emergencias (Farré, 2005; Gil-Calvo, 2003; Kasperson et al., 2003; Palenchar \& Heath, 2007; Pont-Sorribes \& Cortinas-Rovira, 2011).

Los riesgos en el periodismo se han estudiado descriptivamente en Colombia como un indicador de violación a la libertad de prensa que se relaciona con las agresiones contra los periodistas por parte de actores armados ilegales, la fuerza pública y otros agentes del Estado que han afectado la seguridad personal y el desarrollo de la labor informativa imponiendo restricciones (Velásquez, 2003; Roldán, Giraldo \& Flórez, 2003, 2008; Gómez-Giraldo \& Hernández-Rodríguez, 2009; Guerrero, 2010; Fundación para la Libertad de Prensa, 2011, 2012). Sin embargo, y aunque existe dicha literatura, persiste en el ámbito periodístico un vacío de conocimiento sobre los riesgos de seguridad que permitan explicarlo como una variable teórica ligada a la cultura profesional del periodista, de tal manera que tanto la perspectiva descriptiva, como la tradicional sobre comunicación de riesgo, son a menudo criticadas porque descuidan la dimensión científica del riesgo (Vasterman, Scholten \& Ruigrok, 2008).

En ese sentido, con este artículo se pretende aportar empíricamente a dicho campo de conocimiento, tomando como universo poblacional a los periodistas que laboran en Colombia, país considerado como uno de los de mayor riesgo para la seguridad de los periodistas y la libertad de prensa en América Latina (Fundación para la Libertad de Prensa, 2012). El objetivo de esta investigación correlacional-transversal consiste en determinar la relación entre los riesgos de

1 Este artículo forma parte del primer capítulo de resultados de la tesis Autonomía periodística en contextos de violencia social y politica: influencias determinantes de la percepción de autonomía profesional para producir y publicar noticias en Colombia, que se presentó para obtener el doctorado en Comunicación de la Universidad del Norte (Colombia). 
seguridad, pero visto tal fenómeno desde las agresiones recibidas por los periodistas en Colombia y la autonomía profesional, uno de los aspectos más importantes del periodismo y requisito para desarrollar una labor ética y de calidad que sirva a intereses sociales y no particulares (McDevitt, 2006; McQuail, 1992; Weaver, 1998; Bibeny, 2012; Morales, 2007). La investigación responde a tres preguntas relevantes: 1) ¿Qué diferencias existen entre las agresiones recibidas y las características demográficas y laborales de los periodistas en Colombia? 2) ¿Qué factores de la autonomía profesional están asociados a las agresiones recibidas por los periodistas en Colombia? 3) ¿Qué factores de la autonomía profesional predicen el riesgo alto (extremo) de seguridad de los periodistas que laboran en Colombia?

\section{Revisión de la literatura}

La autonomía profesional como factor determinante de la labor periodística

La autonomía periodística es definida como la capacidad de los editores y periodistas de desarrollar su libertad personal usándola de forma responsable para tomar decisiones que beneficien el trabajo periodístico (Gronemeyer, 2002). Los periodistas la relacionan comúnmente con independencia, poder crítico y veracidad (Waisbord, 2013; Splichal \& Sparks, 1994), y es considerada como determinante para asumir roles como el de difusor, adversario y movilizador social que aportan a la construcción de ciudadanía y democracia en los países (Gronemeyer, 2002; Weaver et al., 2006; Mellado, 2014).

La autonomía en el periodismo se ha estudiado empíricamente a través de indicadores como: a) la libertad para informar sobre las historias que el periodista considere relevante, $b$ ) la libertad en su ambiente laboral para seleccionar las historias o noticias en las que trabaja, $c$ ) la libertad para decidir los aspectos de una noticia que deben ser enfatizados, $d$ ) la garantía de que las historias o noticias no sean editadas por otras personas, y e) la libertad para participar de la toma de decisiones editoriales que afecten su condición laboral teniendo en cuenta la relación inseparable entre reportería y edición (Weaver \& Wilhoit, 1996; Mellado \& Humanes, 2012).

Las investigaciones sobre el tema han demostrado, a nivel político, que la autonomía periodística se deteriora en regímenes políticos autoritarios y se eleva en países con sistemas políticos abiertos con mercados de medios más estables y diversos (Stetka \& Örnebring, 2013). Así mismo a nivel organizacional, se ha encontrado que los factores organizativos del medio donde laboran (proce- 
dimientos y rutinas profesionales), la propiedad del medio, el rango editorial y la experiencia profesional son aspectos predictores de la autonomía profesional de los periodistas (Reich \& Hanitzsch, 2013).

En investigaciones comparativas entre países se ha determinado que la autonomía se reduce en contextos con fuertes influencias corporativas y comerciales, correlacionando negativamente con la influencia de las limitaciones de la organización (la propiedad, las expectativas de ganancias y los contratos de publicidad) y las restricciones de influencias externas (empresarios, anunciantes, censura, políticos y gobernantes). Y que en algunos países los bajos niveles percibidos son producto de las presiones que ejercen las élites políticas, y que los altos niveles se asociaban a la percepción de control sobre su labor (Hanitzsch, 2011; Hanitzsch et al., 2012).

De igual manera, a nivel organizacional e individual, se han identificado otros factores diferenciadores como la posición editorial, la ideología política del periodista, la ubicación geográfica del medio de comunicación (Mellado, y Humanes, 2012), el género del periodista, el tipo de medio informativo, y las condiciones laborales, lo que hace comprensible que los niveles más bajos de satisfacción laboral de los periodistas estén asociados a la carencia de autonomía (Weaver \& Wilhoit, 1996; Olson, 1989; Hanitzsch, 2011).

\section{Los riesgos de seguridad en la labor periodística}

Cada año la Fundación para la Libertad de Prensa (FLIP) revela en sus informes los múltiples riesgos de seguridad que enfrentan los periodistas en ejercicio de diversas partes del mundo, los cuales tienen que ver con situaciones de violencia o amenaza, y como tal se definen desde la posibilidad que tiene el periodista de sufrir cualquier tipo de agresión o dańo ocasionado por determinado agente agresor (Freré, 2014; Fundación para la Libertad de Prensa, 2013).

Los riesgos de seguridad son considerados indicadores de violación a la libertad de prensa ya que están asociados a las agresiones contra los periodistas bajo la modalidad de arresto, acoso, violencia, exilio, herido en cubrimiento, atentados, secuestro, amenaza o asesinato, entre otros delitos (Fundación para la Libertad de Prensa, 2011, 2012; Internacional Amnistía, 2012; Martini \& Luchessi, 2004). El Consejo de Derechos Humanos de la Organización para las Naciones Unidas (2012) clasifica los riesgos de seguridad en tres grandes grupos: intimidación, acoso y violencia.

Con relación a esas agresiones, se han documentado cientos de casos de periodistas en el mundo que en contextos hostiles renuncian a su autonomía autocensurándose como mecanismo de supervivencia (Wolton, 1992); en oca- 
siones evitan ahondar o tocar ciertas problemáticas relacionadas con los gobiernos, el poder político, los grupos armados y el narcotráfico; algunos incluso deciden no salir en las cámaras o no firmar sus notas de prensa por temor a ser agredidos, asesinados o exiliados (Manjon-Cabeza, 2012; Arroyave \& Blanco, 2005); otros han padecido de despedidos o agresiones por mantener su autonomía y actitud crítica frente a los gobiernos (Barroeta, 2004; Labio, 2006; Gereda, 2001).

Frente a esta situación, son comprensibles los resultados que indican que los periodistas que laboran en contextos de violencia y de acoso presentan mayores riesgos de padecer trastornos psicológicos que los que trabajan en otros frentes informativos (Feinstein, 2005; Dressing et al., 2007), lo mismo ocurre con los que cubren noticias de narcotráfico y crimen organizado que han presentado puntajes más altos de depresión, ansiedad y estrés postraumático (Flórez, Reyes \& Reidl, 2014).

En el caso de Colombia no es posible estudiar los riesgos de seguridad de los periodistas sin reconocer la incidencia del conflicto armado interno en el contexto social, político, económico y geográfico de la nación en estas últimas cinco décadas de guerra, incluyendo por supuesto su impacto negativo en la libertad de prensa.

Según la Fundación para la Libertad de Prensa (2012), solo entre 2002 y 2012 se reportaron 1419 casos de agresiones a periodistas, y entre 1997 y 2012 hubo 140 periodistas asesinados, una parte por razones del conflicto (violencia política) que da como victimarios y como actores de riesgo para la labor periodística a las guerrillas, los paramilitares, las fuerzas armadas, los narcotraficantes y las bandas criminales.

Los analistas han definido este conflicto armado como un problema geopolítico y económico asociado al poder político, a la expansión terrateniente, a la explotación indebida de los recursos naturales, a la pobreza y a la desigualdad social existente que se empeora por el débil papel del Estado para reducirla. Precisamente, zonas ricas en biodiversidad como Córdoba, el Magdalena Medio, el Meta y el Casanare han sido golpeadas por la pobreza y la violencia por el dominio de élites y la presencia de grupos armados que están allí por los cultivos ilícitos, la renta pública y los recursos mineros o petroleros (González, 2007; Bonilla, 2007; Negrete \& Garcés, 2010).

Esta situación de violencia afecta a los colombianos, pero en especial a las minorías étnicas (afros e indígenas) que, según el informe 2013-2014 de la Defensoría del Pueblo en el Sistema de Alertas Tempranas, son los más golpeados por el conflicto armado. De igual modo, los periodistas han sido también víctimas de agresión por los informes periodísticos que realizan sobre estos problemas estructurales asociados a violaciones de los derechos humanos (Splinder, 2007). 
Algo similar ocurre con los periodistas que informan sobre corrupción, mafias locales u orden público en Colombia, por eso en ocasiones prefieren descartar estos temas o autocensurarse para evitar riesgos contra sus vidas, lo cual afecta a la ciudadanía porque deja de conocer información de interés público vital para sus decisiones diarias (Fundación para la Libertad de Prensa, 2011). En este escenario marcado por la violencia, los periodistas que cubren las noticias en zonas de provincia viven mayores riesgos que los del centro del país o de las ciudades principales (Panqueva, 2012).

\section{Metodología}

El presente estudio cuantitativo se basa en un diseño correlacional-transversal. La población objeto de estudio son los periodistas que laboran en medios informativos de Colombia. Se escogió una muestra por cuotas de 751 periodistas de las tres regiones más pobladas de Colombia: Andina $(n=455)$, Caribe $(n=203)$ y Pacífica ( $\mathrm{n}=93)$, abarcando trece ciudades capitales: en Bogotá se seleccionaron 360 periodistas; en Medellín, 82; en Bucaramanga, 13; en Manizales, 10; en Barranquilla, 82; en Cartagena, 52; en Riohacha, 18; en Santa Marta, 19; en Montería, 9; en Sincelejo, 5; en Valledupar, 8; en Cali, 85; y en Choco, 8.

Para la selección de la muestra se tuvo en cuenta tanto el número de habitantes por región registrado en el último censo nacional oficial (esto debido a que en Colombia no existe un censo de periodistas), como también de acuerdo al tipo de medio informativo donde laboran los periodistas (el 36.6\% labora en prensa, el $32.5 \%$ en radio, el $21.2 \%$ en televisión, el $2.9 \%$ en revistas, el $1.2 \%$ en agencia de noticias y el $4.9 \%$ en medios online).

Para la recolección de información se usó la escala de autonomía profesional que forma parte del cuestionario wjs (Worlds of Journalism Study) creado por investigadores expertos de más de ochenta países de África, Asia, Europa Central, Europa Occidental, Oriente Medio, América del Norte y América Latina, con el fin de analizar las culturas periodísticas a nivel mundial (Hanitzsch et al., 2012). El instrumento se encuentra disponible en la dirección http://www. worldsofjournalism.org/download.htm

Para esta investigación se accedió a la versión en español del instrumento wJS, el cual se complementó con el tema "riesgos de seguridad". Es importante aclarar que este tópico no aparece en el cuestionario oficial en inglés, sino que se añadió con sus respectivos ítems al cuestionario en español adaptado para Colombia y aplicado cara a cara entre junio de 2013 y octubre de 2014, precisamente porque este país padece de un conflicto armado interno, el cual es un factor diferenciador respecto a los demás países participantes del estudio mundial. 
Para operacionalizar los riesgos de seguridad se definió la variable "agresiones recibidas" $(\mathrm{M}=1.333$; $\mathrm{SD}=0.57)$ mediante una escala compuesta unifactorialmente por nueve reactivos: "arresto ilegal" $(\mathrm{M}=1.12$; $\mathrm{DE}=0.52)$, "agresión física" $(\mathrm{M}=1.36 ; \mathrm{DE}=0.79)$, "acoso judicial" $(\mathrm{M}=1.28 ; \mathrm{DE}=0.78)$, "herido en cubrimiento" $(\mathrm{M}=1.28 ; \mathrm{DE}=0.78)$, "amenazas de muerte" $(\mathrm{M}=$ 1.36 ; $\mathrm{DE}=0.89)$, "atentados contra su vida" $(\mathrm{M}=1.12$; $\mathrm{DE}=0.54)$, "secuestro" $(\mathrm{M}=1.11 ; \mathrm{DE}=0.51)$, "agresión verbal" $(\mathrm{M}=2.12 ; \mathrm{DE}=1.29) \mathrm{y}$ "acoso sexual" $(\mathrm{M}=1.17$; $\mathrm{DE}=0.631)$.

Dentro del cuestionario se preguntó a los periodistas sobre las agresiones que han recibido en el ejercicio de su labor informativa, para lo cual ellos respondían a cada reactivo por medio de una escala Likert con puntajes de 1 a 5 ( 1 = nunca; 2 = alguna vez; 3 = a veces; 4 = a menudo; 5 = siempre). Para medir la autonomía profesional se analizaron cinco factores basados en la teoría: 1) "Autonomía para desarrollar el proceso de producción noticiosa", 2) "Autonomía para publicar noticias sobre las élites", 3) "Autonomía para publicar noticias sobre el Estado", 4) "Autonomía para publicar noticias sobre actores armados", y 5) "Autonomía para publicar noticias sobre problemas sociales estructurales".

Los factores fueron evaluados a partir de las respuestas que dieron los periodistas a la pregunta: Qué tanta autonomía tienen para: seleccionar noticias, decidir los aspectos que deben ser enfatizados en las noticias, publicar noticias sobre la élite política, publicar noticias sobre la élite económica, publicar noticias sobre bandas criminales, publicar noticias sobre guerrillas, publicar noticias sobre las minorías étnicas, publicar noticias sobre pobreza, publicar noticias sobre desigualdad socioeconómica, y publicar noticias sobre problemas ambientales.

El primer factor está compuesto por los reactivos: "autonomía para seleccionar noticias" ( $\mathrm{M}=4.25 ; \mathrm{DE}=0.90)$ y "autonomía para decidir los aspectos que deben ser enfatizados en las noticias" $(\mathrm{M}=4.16$; $\mathrm{DE}=0.90)$; el segundo, por los reactivos: "autonomía para publicar noticias sobre la élite política" $(\mathrm{M}=3.76$; $\mathrm{DE}=1.16)$ y "autonomía para publicar noticias sobre la élite económica" $(\mathrm{M}=3.90 ; \mathrm{SD}=1.11)$. El tercero, por los reactivos: "autonomía para publicar noticias sobre bandas criminales" $(\mathrm{M}=3.97$; $\mathrm{DE}=1.16)$ y "autonomía para publicar noticias sobre guerrillas" ( $\mathrm{M}=4.02$; $\mathrm{DE}=1.15)$; y el cuarto, por los reactivos: "autonomía para publicar noticias sobre las minorías étnicas" $(\mathrm{M}=4.18 ; \mathrm{DE}=1.02)$, "autonomía para publicar noticias sobre pobreza" $(\mathrm{M}=$ 4.25 ; $\mathrm{DE}=0.98$ ), "autonomía para publicar noticias sobre desigualdad socioeconómica" $(\mathrm{M}=4.24 ; \mathrm{DE}=1.00)$ y "autonomía para publicar noticias sobre problemas o daños ambientales" $(\mathrm{M}=4.28 ; \mathrm{DE}=1.04)$.

En términos de consistencia interna, el alfa de Cronbach de las subescalas arrojó los siguientes resultados: 0.813 para "agresiones recibidas", 0.848 para "autonomía en el proceso de producción noticiosa”, 0.804 para "autonomía 
en la publicación de información sobre las elites", 0.822 para "autonomía en la publicación de información sobre el Estado", 0.841 para "autonomía en la publicación de información sobre actores armados", y 0.906 para "autonomía en la publicación de información sobre problemas sociales estructurales". Lo que significa que las escalas usadas reportan un buen nivel de fiabilidad, según lo hallado por la literatura científica que reconoce a los alfas entre 0.7 y 0.9 como los más apropiados para hacer investigación básica o aplicada (Cortina, 1993).

Las variables nominales usadas para realizar las pruebas de diferencias de grupos respecto de las agresiones recibidas fueron: sexo (hombre, mujer), sección donde labora (noticias diarias, política internacional, cultura, política nacional, economía, judiciales, deportes, entretenimiento, salud), región (Andina, Caribe y Pacífica), tipo de medio informativo (diario, periódico, revista, televisión, radio, agencia de noticias, medio on line), cobertura del medio informativo (local, regional, nacional, transnacional), propiedad del medio (privado, público, estatal, mixto pero más público, mixto pero más privado), tipo de contrato (definido, indefinido, prestación de servicio, freelance, voluntario, por pauta o cupo, practicante), dedicación laboral (tiempo completo, medio tiempo, freelancer), años de experiencia laboral (entre 0 y 5 años, entre 6 y 11 , entre 12 y 17, entre 18 y 23, entre 24 y 29, más de 29 ańos), cargo (jefe de redacción, editor general, jefe de sección, editor jefe de departamento, productor, reportero, redactor), y nivel educativo de los periodistas (bachiller, técnico, profesional, posgrado).

\section{Resultados}

Riesgos de seguridad: diferencias demográficas y sociolaborales

Dentro de las agresiones más frecuentes contra los periodistas en ejercicio se encontró que el 48.7\% ha sido víctima de agresión verbal, el 20.8\% de agresión física, el $17.7 \%$ ha recibido amenazas, el $14.1 \%$ ha sufrido acoso judicial, el $10.4 \%$ ha sido herido en cubrimiento, el $6.8 \%$ ha sido arrestado ilegalmente, el $8 \%$ ha sido acosado sexualmente, el 5.9\% ha recibido atentados, y el 5.3\% ha sido secuestrado.

Para responder la pregunta sobre las posibles diferencias entre las "agresiones recibidas" y las características demográficas y laborales de los periodistas en Colombia, se consideró oportuno aplicar el test de Kolmogorov-Smirnov para comprobar si los datos de la variable dependiente "agresiones recibidas" tienen distribución normal, y así establecer el procedimiento estadístico adecuado. 
La prueba arrojó que los datos no presentan distribución normal $(\mathrm{Z}=7.708$; $\mathrm{p}=0.000)$, por lo cual se aplicaron pruebas no paramétricas para identificar posibles diferencias con las variables nominales señaladas en el diseño metodológico. Para el caso de la variable demográfica "sexo" que contaba con dos grupos (hombre o mujer) se comprobaron las posibles diferencias con respecto a las "agresiones recibidas" usando la prueba de Mann Whitney; para el resto de variables sociolaborales que tenían tres o más grupos se aplicó la prueba de Kruskall-Wallis, tal como lo sugiere la estadística no paramétrica. La tabla 1 muestra los resultados obtenidos.

Tabla 1. Diferencias entre agresión y las características de los periodistas.

\begin{tabular}{ll}
\hline Variables sociodemográficas y laborales & $p$ \\
\hline Sexo & $0.000^{*}$ \\
Sección informativa & $0.000^{*}$ \\
Región & $0.007^{*}$ \\
Cobertura del medio informativo & 0.294 \\
Propiedad del medio informativo & 0.088 \\
Dedicación laboral & 0.972 \\
Tipo de medio informativo & $0.017^{*}$ \\
Años de experiencia laboral & $0.000^{*}$ \\
Cargo & $0.000^{*}$ \\
Nivel educativo & $0.001^{*}$ \\
Tipo de contrato & 0.380 \\
\hline Nota: Se aplicaron los test de Mann-Whitney y Kruskall-Wallis & \\
$p^{*}<0.05, p^{* *}<0.01$. & \\
Fuente: Elaboración propia. &
\end{tabular}

La tabla 1 muestra que las agresiones contra los periodistas presentan diferencias significativas $(\mathrm{p}<0.05)$ con respecto al sexo, la sección donde labora, la región, el tipo de medio informativo, los ańos de experiencia laboral, el cargo, y el nivel educativo de los periodistas.

Para analizar estas diferencias fue oportuno crear una variable nominal titulada "Niveles de riesgo", esto con el fin de entender cómo operan las agresiones desde lo descriptivo. Los niveles se establecieron desde la clasificación que hace el decreto 4912 dentro del marco del programa de prevención y protección de los derechos a la vida, la integridad y la seguridad de personas, comunidades y grupos en riesgo en Colombia, entre los cuales se encuentran los periodistas que están cobijados por esta legislación.

De esta manera se definió el "nivel de riesgo ordinario" $(43.5 \%, \mathrm{n}=327)$ con aquellos que reportaron que "nunca" han recibido agresión; el "nivel de riesgo extraordinario" $(53.4 \%, \mathrm{n}=401)$ para los que reportaron "alguna vez", 
y el "nivel de riesgo extremo" o alto $(3.1 \%, \mathrm{n}=23)$ para los que reportaron que frecuentemente han recibido agresión, bien sea a "veces", "a menudo" o "siempre". Con este último nivel se complementaron los análisis de forma descriptiva.

En cuanto al sexo, los periodistas hombres presentaron con mayor frecuencia nivel de "riesgo extremo" (77.2\%) en comparación con las mujeres (22.8\%). En cuanto a la sesión donde laboran, las agresiones mostraron diferencias respecto al tipo de noticias, siendo las de nivel de "riesgo extremo" las relacionadas con noticias diarias (47.6\%), política nacional (14.3\%), judiciales $(9.5 \%)$, política general $(9.5 \%)$ y otros $(14.3 \%)$.

En cuanto a lo regional, las frecuencias del nivel de "riesgo extremo" para los periodistas fueron: (52\%) para la región Andina, (39\%) para la región Caribe, y de (9\%) para la región Pacífica (9\%). Entre las ciudades seleccionadas para la muestra, el riesgo alto para los periodistas mostró el siguiente orden: Bogotá, Barranquilla, Cali y Sincelejo.

En cuanto al tipo de medio informativo, los periodistas que laboran en radio presentaron con mayor frecuencia nivel de "riesgo extremo" (43.4\%) en relación con los que trabajan en televisión (21.7\%), diarios (17.3\%), periódicos $(8.7 \%)$ y agencias de noticias $(8.7 \%)$.

En cuanto a los años de experiencia como periodista, el nivel de "riesgo extremo" reportó (26\%) para el rango 0-5 años, (21.7\%) para el 6-11, (4.3\%) para el 12-17, (17.4\%) para el 18-23, (17.4\%) para el 24-29 y (13\%) para los que tienen más de 29 años. En otras palabras, tener menos años de experiencia profesional alcanza con más frecuencia el nivel de "riesgo extremo".

En cuanto al cargo, los periodistas que están en la reportería presentaron con mayor frecuencia nivel de "riesgo extremo" (41\%), y siguieron redactor $(22.7 \%)$, productor $(13.6 \%)$, editor general $(9.1 \%)$ y jefe de redacción $(4.5 \%)$. Mientras que en el rubro de nivel educativo, los periodistas con formación profesional (pregrado y posgrado) mostraron con mayor frecuencia el nivel de "riesgo extremo" (75\%), y enseguida estuvieron los técnicos (20\%) y los bachilleres (5\%).

\section{Autonomía profesional y agresiones recibidas}

Para responder a la pregunta sobre los posibles factores de la autonomía profesional que están asociados a las agresiones contra los periodistas en Colombia, se aplicó la correlación de Spearman, relacionando la variable "agresiones recibidas" y los factores de la autonomía profesional definidos, esto es: "autonomía para informar sobre las élites", "autonomía para informar sobre actores del estado”, 
"autonomía para informar sobre actores armados" y "autonomía para informar sobre problemas sociales estructurales". Los resultados se detallan abajo.

El factor "autonomía para publicar información sobre problemas sociales estructurales" correlaciona positivamente con la variable "agresiones recibidas" $\left(\mathrm{r}=0.073^{*} ; \mathrm{p}<0.05\right)$. Se analizaron los reactivos o ítems de este factor para identificar cuáles de ellos correlacionan específicamente con las agresiones recibidas, y se encontró que a mayor autonomía para informar sobre temas relacionados con pobreza, mayor riesgo de que los periodistas sean acosados judicialmente $\left(\mathrm{r}=0.087^{*} ; \mathrm{p}<0.05\right)$, de recibir amenazas contra su vida $\left(\mathrm{r}=0.087^{*} ; \mathrm{p}<0.05\right)$ o de ser agredido verbalmente $\left(\mathrm{r}=0.080^{*} ; \mathrm{p}<0.05\right)$. Mientras que a mayor autonomía para informar sobre desigualdad social y económica, mayor riesgo de agresión verbal $\left(r=0.077^{*} ; \mathrm{p}<0.05\right)$. Cuando hay mayor autonomía para informar sobre las minorías étnicas, habrá mayor riesgo de ser amenazado $\left(\mathrm{r}=0.085^{*}\right.$; $\mathrm{p}<0.05)$; y a mayor autonomía para informar sobre problemas ambientales, mayor riesgo de agresión verbal $\left(r=0.084^{*} ; \mathrm{p}<0.05\right)$.

Con relación a los factores "autonomía para publicar información sobre los actores armados" y "autonomía para publicar información del Estado", se encontró que la "autonomía para publicar noticias sobre bandas criminales" correlaciona positivamente con el riesgo de ser acosado judicialmente $\left(r=0.076^{*} ; \mathrm{p}<0.05\right)$; "autonomía para publicar noticias sobre el gobierno" correlaciona positivamente con el riesgo de recibir amenazas de muerte $\left(\mathrm{r}=0.076^{*} ; \mathrm{p}<0.05\right)$; "autonomía para publicar noticias sobre las fuerzas armadas" correlaciona positivamente con el riesgo de ser acosado judicialmente $\left(r=0.087^{*}\right.$; $\left.\mathrm{p}<0.05\right)$ o el de recibir amenazas contra su vida $\left(r=0.097^{* *} ; \mathrm{p}<0.01\right)$.

Por otra parte, se identificaron algunas correlaciones negativas en varios reactivos de las variables objeto de estudio, lo que permite inferir que la autonomía profesional de los periodistas no solo es un factor de riesgo para la seguridad de los periodistas, sino también un factor protector en aspectos puntuales del proceso de producción informativa, en donde la "autonomía para seleccionar las noticias" presentó una correlación negativa con el riesgo de arresto ilegal (r $=-0,089 * ; \mathrm{p}<0.05$ ), lo que también ocurrió con la "autonomía para decidir los aspectos que deben ser enfatizados en las noticias" que presentó una correlación negativa con este tipo de agresión $\left(r=-0,074^{*} ; \mathrm{p}<0.05\right)$.

De igual manera, se encontró que la autonomía profesional es un factor protector contra el acoso sexual, pues este tipo de agresión mostró una correlación negativa con la "autonomía para decidir los aspectos que deben ser enfatizados en la noticia" ( $\left.\mathrm{r}=-0.067^{*} ; \mathrm{p}<0.05\right)$, "autonomía para publicar noticias sobre la élite política” ( $\left.\mathrm{r}=-0,092^{* *} ; \mathrm{p}<0.01\right)$, "autonomía para publicar noticias sobre las fuerzas armadas" $\left(\mathrm{r}=-0,084^{*} ; \mathrm{p}<0.05\right)$ y "autonomía para publicar noticias sobre el gobierno" $\left(r=-0,077^{*} ; \mathrm{p}<0.05\right)$. 


\section{Factores de la autonomía profesional predictores del riesgo extremo $o$ alto}

Las correlaciones anteriores se hicieron sin distinción del nivel de riesgo de seguridad de los periodistas, por esta razón se consideró oportuno analizar predictivamente la relación entre autonomía profesional (variable independiente) y la agresión recibida (variable dependiente) seleccionando únicamente los casos de periodistas que reportaron nivel de riesgo extremo (alto).

Para realizar este análisis, se volvió aplicar la prueba Kolmogorov-Smirnov a la variable dependiente "agresiones recibidas" y a los factores de la autonomía profesional que actuaron como variable independiente para estos casos. La prueba mostró que los datos de la variable dependiente se distribuyen normalmente $(\mathrm{Z}=0.766 ; \mathrm{p}=0.601)$, situación similar presentaron los factores: "autonomía para publicar información sobre las élites" $(Z=0.410 ; p=0.996)$, "autonomía para publicar información del Estado" $(Z=0.639 ; \mathrm{p}=0.601)$, "autonomía para publicar información sobre actores armados" $(Z=0.534 ; \mathrm{p}=0.938)$ y "autonomía para publicar información sobre problemas sociales estructurales" $(Z=0.675$; $\mathrm{p}=0.753)$.

Por tal motivo, se procedió a realizar las predicciones mediante un análisis de regresión lineal. La tabla 2 muestra los hallazgos poniendo en conjunto los coeficientes de regresión estandarizados y no estandarizados.

Tabla 2. Factores predictores del riesgo extremo

\begin{tabular}{lccccc}
\hline Factores de la Autonomía & $B$ & error tip. & Beta & $p$ & $R 2$ \\
\hline Autonomía para publicar noticias sobre el Estado & 0.216 & 0.163 & 0.478 & 0.231 & 0.228 \\
Autonomía para publicar noticias sobre los actores armados & 0.094 & 0.118 & 0.308 & 0.459 & 0.095 \\
Autonomía para publicar noticias sobre las élites & 0.242 & 0.055 & $0.875^{* *}$ & 0.004 & 0.726 \\
Autonomía para publicar noticias sobre los problemas & 0.256 & 0.072 & $0.825^{*}$ & 0.012 & 0.658 \\
sociales estructurales & & & & & \\
\hline $\mathrm{p}^{*}<0.05, \mathrm{p}^{* *}<0.01$. & & & & & \\
Fuente: Elaboración propia. & & & &
\end{tabular}

La tabla 2 revela que la "autonomía para publicar noticias sobre élites" y "autonomía para publicar noticias sobre problemas sociales estructurales" son los mayores predictores de las "agresiones recibidas" contra los periodistas en nivel de riesgo alto o extremo, no solo porque las variables tienen una correlación alta en sentido positivo, sino también porque las "agresiones recibidas" se explican desde un $72.6 \%$ de la varianza de la categoría "autonomía para publicar noticias sobre las élites" (élites políticas y económicas) y un $65.8 \%$ de la varianza de la categoría "autonomía para publicar noticias sobre problemas 
sociales estructurales" (situación de las minorías étnicas, pobreza, desigualdad socioeconómica y problemas ambientales), lo que demuestra que estos dos factores influyen en la configuración del riesgo alto de seguridad de los periodistas en Colombia.

\section{Discusión y conclusiones}

Los riesgos de seguridad que enfrentan los periodistas en Colombia son diversos y están asociados con las distintas agresiones señaladas en los informes de la Fundación para la Libertad de Prensa. En este estudio se encontró que la agresión verbal, la agresión física, las amenazas y el acoso judicial son los riesgos más frecuentes que los periodistas asumen durante su labor.

Las agresiones recibidas a nivel general presentaron diferencias significativas con respecto al sexo, la sección informativa, los años de experiencia laboral, el cargo, el nivel educativo del periodista, el tipo de medio informativo, y la región, que es un aspecto señalado en otros estudios como un factor diferenciador del riesgo (Panqueva, 2012). No obstante, quedó sin explorar cómo operan las diferencias entre lo citadino y lo provincial porque solo se encuestó a periodistas de ciudades capitales, lo cual fue una limitación de esta investigación.

Se encontró que la autonomía para publicar noticias sobre gobiernos y fuerzas armadas correlaciona en sentido positivo con el riesgo que tiene el periodista de recibir acoso judicial y/o amenazas de muerte, lo cual es congruente con los análisis de Labio (2006), Barroeta (2004) y Gereda (2001), quienes han identificado en diversos países casos de periodistas agredidos y afectados en sus derechos laborales por criticar las malas actuaciones o decisiones de los gobernantes, lo que hace que la autonomía desde este énfasis noticioso sea un factor de riesgo para los periodistas y un indicador político preocupante, si se tiene en cuenta que la labor constitucional de los agentes del Estado es garantizar la libertad de prensa y proteger a los ciudadanos, no el de agredirlos o vulnerarles sus derechos fundamentales.

La autonomía para publicar noticias sobre bandas criminales correlacionó positivamente con las agresiones recibidas. Dicho hallazgo también es sobresaliente en los trabajos de Manjon-Cabeza (2012) Hughes (2006) y Arroyave \& Blanco (2005), autores que refieren las censuras, temores y violencia que padecen los periodistas en diversos países al cubrir temas relacionados con actores armados ilegales y narcotráfico.

De igual manera, la autonomía para publicar noticias sobre problemas sociales estructurales (pobreza, situación de las minorías étnicas, desigualdad 
social y problemas ambientales) mostró ser un factor de riesgo para la seguridad de los periodistas en Colombia, al correlacionar positivamente con las agresiones recibidas.

En efecto, González (2007) y Bonilla (2007) consideran que estos problemas han recrudecido el conflicto armado colombiano, lo que hace comprensible que los periodistas que cubren este tipo de temas asociados con violación de los derechos humanos, sean los más afectados en su seguridad, tal como lo señalan los análisis de Splinder (2007).

Adicionalmente se encontró como nuevo hallazgo, que la autonomía profesional es un factor de protección contra el acoso sexual, al correlacionar en sentido negativo con la autonomía para decidir los aspectos que deben ser enfatizados en la noticia y para publicar noticias sobre la élite política, las fuerzas armadas y el gobierno. Así mismo mostró ser un factor preventivo del arresto ilegal al correlacionar negativamente con la autonomía para decidir los énfasis en la noticia.

Los bajos coeficientes de correlación son explicados no solo por el carácter multicausal que rodea los fenómenos sociales, sino también porque las correlaciones se analizaron sin distinguir los niveles de riesgo, de tal forma que al seleccionar a los periodistas en riesgo extremo las correlaciones entre la autonomía profesional y las agresiones fueron altas, aspecto en el que los mayores predictores fueron la autonomía para publicar noticias sobre problemas sociales estructurales y sobre las élites (políticas y económicas), grupos influyentes en los medios informativos (Hanitzsch, 2011; Hanitzsch et al., 2012), que cuando se les aborda o critica periodísticamente se ha encontrado que aumentan los riesgos de seguridad de los periodistas (Manjon-Cabeza, 2012; Guerrero, 2010; Roldán, Giraldo \& Flórez, 2008; Gómez-Giraldo \& Hernández-Rodríguez, 2009; Arroyave \& Blanco, 2005).

En términos generales los resultados demuestran que en Colombia existe un perfil del periodista de riesgo, de tal forma que los que enfrentan mayores riesgos de seguridad se caracterizan por: ser hombres, laborar en la radio, cubrir noticias diarias en calidad de reporteros, ser profesionales con una experiencia que oscila entre 0 y 11 años en el oficio, y abordar con autonomía profesional temas periodísticos relativos a problemas sociales estructurales y a las actuaciones de las élites del poder político y económico de este país.

\section{Bibliografía}

Arroyave, J. \& Blanco, I. (2005). Cómo perciben los periodistas su profesión: entre el agotamiento y la fascinación. Investigación y Desarrollo, 13(2), 364-389. 
Barroeta, E. (2004). El riesgo de informar. En Petruvska, S. (Ed.). Periodistas en la mira (pp. 104-112). Caracas: Alfa.

Bilbeny, N. (2012). Ética del Periodismo. Barcelona: Edicions Universitat.

Bonilla, L. (2007). Magdalena Medio: de las luchas por la tierra a la consolidación de autoritarismos subnacionales. En Romero M. (Ed.). Parapolítica: la ruta de la expansión paramilitar y los acuerdos políticos (pp. 341-392). Bogotá: Corporación Nuevo Arco Iris/Intermedio Editores.

Canel, M. \& Sádaba, T. (1999). La investigación académica sobre las actitudes profesionales de los periodistas. Una descripción del estado de la cuestión. Comunicación y Sociedad, 12(2), 9-32.

Consejo de Derechos Humanos de la Organización para las Naciones Unidas, (2012). Tema 3 de la agenda Promoción y protección de todos los derechos humanos, civiles, políticos, económicos, sociales y culturales, incluido el derecho al desarrollo (GE.12-17176 -S- 260912). Nueva York: onU. Recuperado de http://ap.ohchr.org/documents/S/HRC/d_res_dec /A_HRC_21_L6.pdf

Cortina, J. (1993). What is Coefficient Alpha? An Examination of Theory and Applications. Journal of Applied Psychology, 78(1), 98-104.

Dressing, H., Martini, M., Witthoft, M., Bailer, J. \& Gass, P. (2007). Are Journalists more Frequently Victims of Stalking? Results of First Empirical Examinations. Gesundheitswesen, 69(12), 699-703.

Farré, J. (2005). Comunicación de riesgo y espirales del miedo. Nueva Época, (3), 95-119.

Freré, M.-S. (2014). Journalist in Africa: A High-Risk Profession under Threat. Journal of African Media Studies, 2(6), 181-198.

Feinstein, A. \& Nicolson, D. (2005). Embedded Journalists in the Iraq War: ¿Are they at Greater Psychological Risk? Journal of Traumatic Stress, 18(2), 129-132.

Fundación para la Libertad de Prensa (2013). Manual de autoprotección para periodistas. Bogotá: FLIP. Recuperado de http://flip.org.co/es/content/manual-de-autoprotecci\%C3\%B3n -para-periodistas

Fundación para la Libertad de Prensa (2012). Informe sobre el estado de la libertad de prensa en Colombia: De las balas a los expedientes. Bogotá: FLIP. Recuperado de http://flip.org.co /resources/documents/informe-2012.pdf 
Fundación para la Libertad de Prensa (2011). Informe sobre el estado de la libertad de prensa en Colombia: ¿La censura en las regiones llegó para quedarse? Bogotá: FLIP. Recuperado de http:// flip.org.co/resources/documents/informe-2011.pdf

Flores R., Reyes, V. \& Reidl, L. (2014). El impacto psicológico de la guerra contra el narcotráfico en periodistas mexicanos. Revista Colombiana de Psicología, 23(1), 177-193.

Gereda, S. (2001). Riesgos del periodista al investigar corrupción de dictaduras militares. Razón y Palabra, (22). Recuperado de http://www.razonypalabra.org.mx/anteriores/n22 122_sgereda.html

Gil-Calvo, E. (2003). El miedo es el mensaje: riesgo, incertidumbre y medios de comunicación. Madrid: Alianza.

Gómez-Giraldo, J. \& Hernández-Rodríguez, J. (2009). Libertad de prensa en Colombia: entre la amenaza y la manipulación. Palabra Clave, 12(1), 13-35.

González, J. (2007). Los paramilitares y el colapso estatal en Meta y Casanare. En Romero, M. (Ed.). Parapolitica: la ruta de la expansión paramilitar y los acuerdos politicos (pp. 239-284). Bogotá: Intermedio Editores.

Gronemeyer, M. (2002). Periodistas chilenos: El reto de formar profesionales autónomos e independientes. Cuadernos de Información, (15), 53-70.

Guerrero, A. (2010). País lejano y silenciado: autocensuras y prácticas periodísticas en el periodismo regional. Bogotá: Fundación para la Libertad de Prensa.

Hanitzsch, T. (2011). Populist Disseminators, Detached Watchdogs, Critical Change Agents and Opportunist Facilitators: Professional Milieus, the Journalistic Field and Autonomy in 18 Countries. The International Communication Gazette, (73), 477-494.

Hanitzsch, T., Seethaler, J., Skewes, E., Anikina, M., Berganza, R., Cangöz, I. et al. (2012). Worlds of Journalism: Journalistic Cultures, Professional Autonomy and Perceived Influences across 18 Nations. En Weaver, D. \& Willnat, L. (Eds.). The Global Journalist in the 21st Century (pp. 473-494). Nueva York: Routledge.

Hughes, S. (2006). Newsrooms in Conflict: Journalism and the Democratization of Mexico. USA: University of Pittsburgh.

Internacional Amnistía (2012). Informe Anual 2012 de Amnistía Internacional. El estado de los derechos humanos en el mundo. España: Amnistía Internacional. Recuperado de http://files .amnesty.org/air12/air_2012_full_es.pdf 
Kasperson, J., Kasperson, R., Pidgeon, N. \& Slovic, P. (2003). The Social Amplification of Risk: Assessing Fifteen Years of Research and Theory. En Pidgeon, D., Kasperson, R. \& Slovic, P. (Eds.). The Social Amplification of Risk (pp. 13-46). Cambridge: Cambridge University Press.

Labio, A. (2006). Comunicación, periodismo y control informativo: Estados Unidos, Europa y España. Barcelona: Anthropos.

Manjon-Cabeza, A. (2012). La solución: La legalización de las drogas. España: Penguin Random House.

Martini, S. \& Luchessi, L. (2004). Los que hacen la noticia: periodismo, información y poder. Buenos Aires: Biblos.

McDevitt, M. (2006). In Defense of Autonomy: A Critique of the Public Journalism Critique. Journal of Communication, 53(1), 155-164.

McQuail, D. (1992). Media Performance: Mass Communication and the Public Interest. Londres: Sage.

Mellado, C. (2014). Professional Roles in News: Six Dimensions of Journalistic Role Performance. Journalism Studies. Recuperado de http://www.tandfonline.com/doi/full/10.1080 /1461670X.2014.922276\#.VHXru3Nd3NUMellado, C. \& Humanes, M. (2012). Modeling perceived profesional autonomy in Chilean Journalism. Journalism, 13(8), 985-1003.

Miralles, A. (2009). Periodismo público en la gestión del riesgo. Lima: Comunidad Andina.

Morales, O. (2007). Periodismo: Ética y Paz. Cali: Universidad del Valle.

Negrete, V. \& Garcés, M. (2010). Análisis sociopolitico de Montería y propuestas sobre liderazgo, participación y compromiso ciudadano. Montería: Fundación del Sinú.

Obregón, R, Arroyave, J. \& Barrios, M. (2010). Periodismo y comunicación para la gestión de riesgo en la subregión andina: discursos periodísticos y perspectivas para un enfoque prospectivo y preventivo. Folios, (23), 105-135.

Obregón, R., Arroyave, J. \& Barrios, M. (2009). Cubrimiento periodístico de la gestión del riesgo en la subregión Andina: discursos periodísticos y perspectivas desde la comunicación para el cambio social. Lima: Comunidad Andina/CAPRADE.

Olson, L. (1989). Job Satisfaction of Journalists and PR Personnel. Public Relations Review, 15(4), 37-45. 
Ortega, F., \& Humanes, M. (2000). Algo más que periodistas. Sociología de una profesión. Barcelona: Ariel.

Palenchar, M. \& Heath, R. (2007). Strategic Risk Communication: Adding Value to Society. Public Relations Review, 33(2), 120-129.

Panqueva, L. (2012). ¿Qué pasa cuando amenazan a un periodista colombiano? Bogotá: Universidad del Rosario. Recuperado de http://www.urosario.edu.co/urosario_files/8c/8ccbea1a -7df1-4ae3-97c0-a7bedfd3dd43.pdf

Pont-Sorribes, C. \& Cortinas-Rovira, S. (2011). Journalistic Practice in Risk and Crisis Situations: Significant Examples from Spain. Journalism, 12(8), 1052-1066.

Roldán, I., Giraldo, D. \& Flórez, M. (2008). Impacto del conflicto colombiano en los periodistas. Revista Colombiana de Psiquiatría, 37(1), 66-80.

Roldán, I., Giraldo, D. \& Flórez, M. (2003). Periodistas, Guerra y Terrorismo. Bogotá: Planeta.

Reich, Z. \& Hanitzsch, T. (2013). Determinants of Journalists' Professional Autonomy: Individual and National Level Factors Matter more than Organizational Ones. Mass Communication and Society, 16(1), 133-156.

Splinder, W. (2007). Los periodistas colombianos frente al conflicto armado. En Fraguas J. (Ed.), Periodismo preventivo: otra manera de informar sobre las crisis y los conflictos internacionales (pp.75-81). Madrid: Los Libros de la Catarata.

Splichal, S. \& Sparks, C. (1994). Journalists for the 21st Century. Tendencies of Professionalization among First-Year Students in 22 Countries. New Jersey: Ablex Pub.

Stetka, V. \& Örnebring, H. (2013). Investigative Journalism in Central and Eastern Europe: Autonomy, Business Models, and Democratic Roles. International Journal of Press-Politics, $18(4), 413-435$.

Vasterman, P., Scholten, O. \& Ruigrok, N. (2008). A model for Evaluating Risk Reporting - The Case of umTs and Fine Particles. European Journal of Communication, 23(3), 319-341.

Velásquez, C. (2003). El estado de la libertad de prensa en Colombia: una mirada con énfasis en las regiones. Palabra Clave, (8), 1-16.

Waisbord, S. (2013). Reinventing Professionalism.Cambridge: John Wiley \& Sons. 
Weaver, D., Beam, R., Brownlee, B., Voakes, P. \& Wilhoit, G. (2006). The American Journalist in the 21st Century: U.S. News People at the Dawn of a New Millennium. Nueva York: Routledge.

Weaver, D. (1998). The Global Journalist: News People Around The World. Cresskill. New Jersey: Hampton Press.

Weaver, D. \& Wilhoit, G. (1996). The American Journalist in the 1990s: U.S. Newspeople at Theend of an Era. New Jersey: Psychology Press.

Wolton, D. (1992). War game: la información y la guerra. París: Siglo xxi.

Recibido el 9 de enero de 2015. Aceptado el 20 de octubre de 2015. 